Louisiana State University

LSU Digital Commons

$1-1-2017$

\title{
Observations of the first electromagnetic counterpart to a gravitational wave source by the TOROS collaboration
}

\author{
Lucas M. MacRi \\ Texas A\&M University \\ Mario C. Dlaz \\ University of Texas Rio Grande Valley \\ Diego Garcia Lambas \\ Observatorio Astronomico de la Universidad Nacional de Cordoba \\ G. González \\ R. Hynes
}

Follow this and additional works at: https://digitalcommons.Isu.edu/physics_astronomy_pubs

\section{Recommended Citation}

MacRi, L., Dlaz, M., Lambas, D., González, G., \& Hynes, R. (2017). Observations of the first electromagnetic counterpart to a gravitational wave source by the TOROS collaboration. Proceedings of the International Astronomical Union, 13 (S338), 80-83. https://doi.org/10.1017/S1743921318003630

This Conference Proceeding is brought to you for free and open access by the Department of Physics \& Astronomy at LSU Digital Commons. It has been accepted for inclusion in Faculty Publications by an authorized administrator of LSU Digital Commons. For more information, please contact ir@lsu.edu. 


\section{University of Texas Rio Grande Valley \\ ScholarWorks @ UTRGV}

$10-20-2017$

\section{Observations of the First Electromagnetic Counterpart to a Gravitational-wave Source by the TOROS Collaboration}

M. C. Diaz

L. M. Macri

D. Garcia Lambas

C. Mendes De Oliveira

J. L.Nilo Castellón

See next page for additional authors

Follow this and additional works at: https://scholarworks.utrgv.edu/pa_fac

Part of the Astrophysics and Astronomy Commons

\section{Recommended Citation}

M. C. Díaz, et. al., (2017) Observations of the First Electromagnetic Counterpart to a Gravitational-wave Source by the TOROS Collaboration.Astrophysical Journal Letters848:2. DOI: http://doi.org/10.3847/ 2041-8213/aa9060

This Article is brought to you for free and open access by the College of Sciences at ScholarWorks @ UTRGV. It has been accepted for inclusion in Physics and Astronomy Faculty Publications and Presentations by an authorized administrator of ScholarWorks @ UTRGV. For more information, please contact justin.white@utrgv.edu, william.flores01@utrgv.edu. 


\section{Authors}

M. C. Diaz, L. M. Macri, D. Garcia Lambas, C. Mendes De Oliveira, J. L.Nilo Castellón, T. Ribeiro, B. Sanchez, W. Schoenell, L. R. Abramo, S. Akras, J. S. Alcaniz, Rodolfo Artola, M. Beroiz, S. Bonoli, J. Cabral, R. Camuccio, M. Castillo, V. Chavushyan, P. Coelho, C. Colazo, M. V. Costa-Duarte, H. Cuevas Larenas, D. L. Depoy, M. Domínguez Romero, D. Dultzin, D. Fernández, J. García, and C. Girardini 


\title{
Observations of the First Electromagnetic Counterpart to a Gravitational-wave Source by the TOROS Collaboration
}

\author{
M. C. Díaz (1D, L. M. Macri ${ }^{1}$ (D), D. Garcia Lambas ${ }^{3}$, C. Mendes de Oliveira ${ }^{4}$, J. L. Nilo Castellón ${ }^{5,6}$, T. Ribeiro ${ }^{7}$, B. Sánchez ${ }^{3}$, \\ W. Schoenell ${ }^{4,8}$, L. R. Abramo ${ }^{9}$, S. Akras $^{10}$, J. S. Alcaniz ${ }^{10}$, R. Artola ${ }^{3}$, M. Beroiz ${ }^{1}$, S. Bonoli ${ }^{11}$, J. Cabral ${ }^{3}$, R. Camuccio ${ }^{1}$, \\ M. Castillo ${ }^{1}$, V. Chavushyan ${ }^{12}$ (D), P. Coelho ${ }^{4}$, C. Colazo ${ }^{3}$, M. V. Costa-Duarte ${ }^{4}$, H. Cuevas Larenas ${ }^{6}$, D. L. DePoy ${ }^{2}$, \\ M. Domínguez Romero ${ }^{3}$, D. Dultzin ${ }^{13}$ (D), D. Fernández ${ }^{14}$, J. García ${ }^{1}$, C. Girardini ${ }^{3}$, D. R. Gonçalves ${ }^{15}$, T. S. Gonçalves ${ }^{15}$, \\ S. Gurovich ${ }^{3}$, Y. Jiménez-Teja ${ }^{10}$, A. Kanaan ${ }^{8}$, M. Lares $^{3}$, R. Lopes de Oliveira ${ }^{7,16}$, O. López-Cruz ${ }^{12}$ (iD), J. L. Marshall ${ }^{2}$ (D), R. Melia ${ }^{3}$, \\ A. Molino ${ }^{4}$, N. Padilla ${ }^{14}$ (D) T. Peñuela ${ }^{1,17}$, V. M. Placco ${ }^{18,19}$, C. Quiñones ${ }^{3}$ (D), A. Ramírez Rivera ${ }^{6}$, V. Renzi $^{3}$, L. Riguccini ${ }^{15}$, \\ E. Ríos-López ${ }^{12}$ (i) , H. Rodriguez ${ }^{3}$, L. Sampedro ${ }^{4}$, M. Schneiter ${ }^{3}$, L. Sodré ${ }^{4}$, M. Starck ${ }^{3}$, S. Torres-Flores ${ }^{6}$, \\ M. Tornatore ${ }^{3}$, and A. Zadrożny ${ }^{1}$ \\ ${ }^{1}$ Center for Gravitational Wave Astronomy and Department of Physics \& Astronomy, University of Texas—Río Grande Valley, Brownsville, TX, USA \\ ${ }^{2}$ George P. and Cynthia W. Mitchell Institute for Fundamental Physics \& Astronomy, Department of Physics \& Astronomy, \\ Texas A\&M University, College Station, TX, USA; 1macri@tamu.edu \\ ${ }^{3}$ IATE-OAC, Universidad Nacional de Córdoba-CONICET, Córdoba, Argentina \\ ${ }^{4}$ Instituto de Astronomia, Geofísica e Ciências Atmosféricas da U. de São Paulo, São Paulo, SP, Brazil \\ ${ }^{5}$ Instituto de Investigación Multidisciplinario en Ciencia y Tecnología, Universidad de La Serena, La Serena, Chile \\ ${ }_{7}^{6}$ Departamento de Física y Astronomía, Universidad de La Serena, La Serena, Chile \\ ${ }^{7}$ Departamento de Física, Universidade Federal de Sergipe, São Cristóvão, SE, Brazil \\ ${ }^{8}$ Departamento de Física, Universidade Federal de Santa Catarina, Florianópolis, SC, Brazil \\ ${ }^{9}$ Departamento de Física Matemática, Instituto de Física, Universidade de São Paulo, São Paulo, SP, Brazil \\ ${ }^{10}$ Departamento de Astronomia, Observatório Nacional, Rio de Janeiro, RJ, Brazil \\ ${ }^{11}$ Centro de Estudios de Física del Cosmos de Aragón, Teruel, Spain \\ ${ }^{12}$ Instituto Nacional de Astrofísica, Óptica y Electrónica, Tonantzintla, Puebla, México \\ ${ }^{13}$ Instituto de Astronomía, Universidad Nacional Autónoma de México, Ciudad de México, México \\ ${ }^{14}$ Instituto de Astrofísica, Pontificia Universidad Católica de Chile, Santiago, Chile \\ ${ }^{15}$ Observatorio do Valongo, Universidade Federal do Rio de Janeiro, Río de Janeiro, RJ, Brazil \\ ${ }^{16}$ X-ray Astrophysics Laboratory and CRESST, NASA Goddard Space Flight Center, Greenbelt, MD, USA \\ ${ }^{17}$ Ludwig Maximilian Universität Munich, Faculty of Physics, Munich, Germany \\ ${ }^{18}$ Department of Physics, University of Notre Dame, Notre Dame, IN, USA \\ 19 Joint Institute for Nuclear Astrophysics-Center for the Evolution of the Elements, USA \\ Received 2017 September 29; revised 2017 October 1; accepted 2017 October 1; published 2017 October 16
}

\begin{abstract}
We present the results of prompt optical follow-up of the electromagnetic counterpart of the gravitational-wave event GW170817 by the Transient Optical Robotic Observatory of the South Collaboration. We detected highly significant dimming in the light curves of the counterpart $(\Delta g=0.17 \pm 0.03 \mathrm{mag}, \Delta r=0.14 \pm 0.02 \mathrm{mag}$, $\Delta i=0.10 \pm 0.03 \mathrm{mag}$ ) over the course of only 80 minutes of observations obtained $\sim 35 \mathrm{hr}$ after the trigger with the T80-South telescope. A second epoch of observations, obtained $\sim 59 \mathrm{hr}$ after the event with the EABA $1.5 \mathrm{~m}$ telescope, confirms the fast fading nature of the transient. The observed colors of the counterpart suggest that this event was a "blue kilonova" relatively free of lanthanides.
\end{abstract}

Key words: gamma-ray burst: individual (170817A) - stars: neutron

Supporting material: machine-readable table

\section{Introduction}

The network of advanced ground-based gravitational-wave (GW) interferometers constituted by the Advanced Laser Interferometer Gravitational-wave Observatory (LIGO; LIGO Scientific Collaboration 2015, hereafter, "LSC") started its second observational campaign (O2) on 2016 November 30. On 2017 August 1, Advanced Virgo (Acernese et al. 2015) began its first observational campaign, initiating the first concurrent monitoring of the sky by a network of three GW interferometers. $^{20}$

The first detection of a binary black hole $(\mathrm{BBH})$ merger by Advanced LIGO opened the era of GW astronomy (GW150914; Abbott et al. 2016a). Three similar events have been detected since then, two by Advanced LIGO and the most recent one by

\footnotetext{
$\overline{20}$ This concurrent campaign ended on 2017 August 25, http://www.ligo.org/ news/index.php\#O2end.
}

Advanced LIGO/Virgo (GW151226, GW170104, and GW170814; Abbott et al. 2016b, 2017a, 2017b). For many years, particularly since the discovery of the binary pulsar PSR B1913 +16 by Hulse \& Taylor (1975) and the evidence for energy loss in this system as expected from GW emission (Taylor \& Weisberg 1982), binary neutron star (BNS) mergers were anticipated to be one of the main sources found by advanced GW detectors. Hence, it was surprising that the first four $\mathrm{GW}$ detections were BBHs.

The LSC and the Virgo Collaboration (VC) issued on 2013 June 6 a worldwide call to participate in electromagnetic (EM) and multi-messenger observations of $\mathrm{GW}$ events recorded by their detectors, using a wide range of telescopes and instruments of "mainstream astronomy." 21 The Transient Optical Robotic Observatory of the South Collaboration

\footnotetext{
${ }^{21}$ http://www.ligo.org/scientists/GWEMalerts.php
} 
(TOROS; Díaz et al. 2014; Benacquista et al. 2014) was organized in 2013 to participate in these observations. While seeking to deploy a wide-field optical telescope on Cordón Macón in north-west Argentina (Renzi et al. 2009; Tremblin et al. 2012), the collaboration has been utilizing other resources for follow-up activities. Our activities during LIGO's first observational campaign (O1) have been reported by Colazo et al. (2015) and Díaz et al. (2016).

On 2017 August 17 12:41:04 UTC (BJD 2457983.02857), a BNS merger candidate was identified in data from the LIGO Hanford (H1) detector (LIGO/Virgo G298048, LIGO Scientific Collaboration \& Virgo Collaboration 2017a, 2017b). The Gamma-ray Burst Monitor (GBM) on board Fermi (Bissaldi et al. 2009) detected an event $\sim 2$ s after the GW trigger, which was given the designation GRB170817A (Connaughton et al. 2017; Goldstein et al. 2017; von Kienlin et al. 2017). The significance of the GW detection was initially estimated as an equivalent false-alarm rate of 1 -in- $10^{4}$ years based on the $\mathrm{H} 1$ data alone. The effective distance was estimated as $\sim 58 \mathrm{Mpc}$, and the initial localization estimate, based only on $\mathrm{H} 1$ data, was quite broad. LIGO Scientific Collaboration \& Virgo Collaboration (2017b) reported that the GW event was also clearly visible in data from LIGO Livingston (L1), although there was a coincident noise artifact. Further analysis of GW data from all detectors (including Advanced Virgo) provided better estimates of the localization probability and of the luminosity distance, $40 \pm 8 \mathrm{Mpc}$ (LIGO Scientific Collaboration \& Virgo Collaboration 2017c, 2017d, 2017e).

At the time of the event, the horizon ranges (the maximum distance at which a BNS merger could be detected with S/N > 8; Finn \& Chernoff 1993; Allen et al. 2012; Chen et al. 2017) were 218, 107, and $58 \mathrm{Mpc}$ for L1, H1, and Virgo, respectively (Abbott et al. 2017b). The GW trigger has subsequently been confirmed as a very high-confidence detection consistent with a BNS merger, and given the designation GW170817 (LIGO Scientific Collaboration \& Virgo Collaboration 2017f).

About $11 \mathrm{hr}$ after the GW trigger, several groups participating in the aforementioned GW/EM collaboration reported (Allam et al. 2017; Coulter et al. 2017; Yang et al. 2017) the detection of a putative EM counterpart (initially called SSS17a or DLT17ck, official IAU name AT2017gfo; hereafter, "the transient") located $10^{\prime \prime}$ from the center of NGC 4993, an S0 galaxy at a distance of $38 \pm 5 \mathrm{Mpc}$ (Kourkchi \& Tully 2017). Its J2000 coordinates were reported as R.A. $=13^{\mathrm{h}} 09^{\mathrm{m}} 48^{\mathrm{s}} .1$, decl. $=-23^{\mathrm{d}} 22^{\mathrm{m}} 53^{\mathrm{s}}$ (Coulter et al. 2017, and D. A. Coulter et al. 2017, in preparation).

\section{Observations and Photometry}

\subsection{Observations}

The TOROS Collaboration participated in the search for the EM counterpart of GW170817 starting just a few hours after the trigger. On the nights of 2017 August 17 and 18, we surveyed 26 nearby galaxies contained in the initial localization region using two facilities: the T80-South telescope (described below) and a Meade LX200 16-inch telescope equipped with a SBIG STF 8300 camera, located in Tolar Grande, Argentina (Díaz et al. 2017a, 2017b). Once the candidate counterpart was identified near NGC 4993 (see the references above), we focused our efforts on that source.
On 2017 August 18 ( $\sim 35 \mathrm{hr}$ after the GW trigger $)$ we observed the transient using the T80-South ${ }^{22}$ telescope (T80S; C. Mendes de Oliveira et al. 2017, in preparation) located at CTIO. This telescope has a primary mirror with a diameter of $0.83 \mathrm{~m}$ and a camera equipped with an E2V CCD290-99 detector, consisting of $9216 \times 9232$ pixels with a plate scale of 0 . $55 /$ pix that yields a field of view (FoV) 1.4 on a side. We obtained 16, 15, and 15 one-minute exposures through SDSS $g$, $r$, and $i$ filters, respectively, at airmass values from 1.35 to 1.97 over the course of 80 minutes. The typical seeing was $\sim 1$." 8 . The left panel of Figure 1 shows a color composite of a small subsection (9.5 on a side) of the T80S field of view (FoV) centered on the transient.

On 2017 August 19 ( 59 hr after the GW trigger $)$ we imaged the same source using the $1.54 \mathrm{~m}$ telescope located at the Estación Astrofísica de Bosque Alegre (EABA) and an Apogee ALTA F16 camera equipped with a KAF-16083 sensor, consisting of $4096 \times 4096$ pixels with a plate scale of 0 .'24/ pix that yields a FoV of $17^{\prime}$ on a side. We obtained 88 one-minute unfiltered exposures in $2 \times 2$ binned mode to expedite readout and match the seeing $(\sim 3 . \prime 5)$.

\subsection{Photometry}

Our photometry is based on the observations obtained with the T80S and the EABA $1.5 \mathrm{~m}$ telescopes. We used the IRAF ${ }^{23}$ CCDPROC package to debias and flat-field the raw frames. We carried out time-series point-spread function (PSF) photometry using DAOPHOT/ALLSTAR (Stetson 1987), ALLFRAME (Stetson 1994), and related programs, kindly provided by P. Stetson. The steps we performed closely follow those outlined in Macri et al. (2006, 2015). We modeled the PSFs by fitting a Moffat function with $\beta=2.5$ to $25-50$ bright and isolated stars in each image. The fitting radii were 4 and 6 pix, and the PSFs were defined out to 8 and 10 pix for T80S and EABA, respectively. The local background level for each star was determined using annuli from $8-10$ and $10-15$ pix for T80S and EABA, respectively.

We first used DAOPHOT to detect sources in each image with a significance of $4 \sigma$ or greater, identify bright isolated stars, and determine the PSFs. We then used ALLSTAR to obtain preliminary PSF photometry for all of the sources. Next, we used DAOMATCH and DAOMASTER to derive robust geometric transformations between all of the images obtained with a given telescope and filter. We generated four "master frames" (one for each telescope and filter) by median-combining a subset of images with low background values and good seeing.

Given the close proximity of the transient to its host galaxy, we used the IMFIT package (Erwin 2015) to model and subtract its light distribution from each image. We first determined the best-fit parameters on the master frames, masking all of the stars within $30^{\prime \prime}$ of the galaxy center as well as its innermost $5^{\prime \prime}$ and fitting the intervening region using Sérsic profiles. Once the best-fit parameters (ellipticity, position angle, Sérsic index, effective radius) for a given band were determined from the master image, they were held fixed in the fitting process for each individual frame. We only allowed the intensity scaling to remain a free parameter, since the location

\footnotetext{
22 http://www.splus.iag.usp.br/t80s-telescope

23 IRAF is distributed by the National Optical Astronomy Observatory, which is operated by the Association of Universities for Research in Astronomy (AURA) under cooperative agreement with the National Science Foundation.
} 


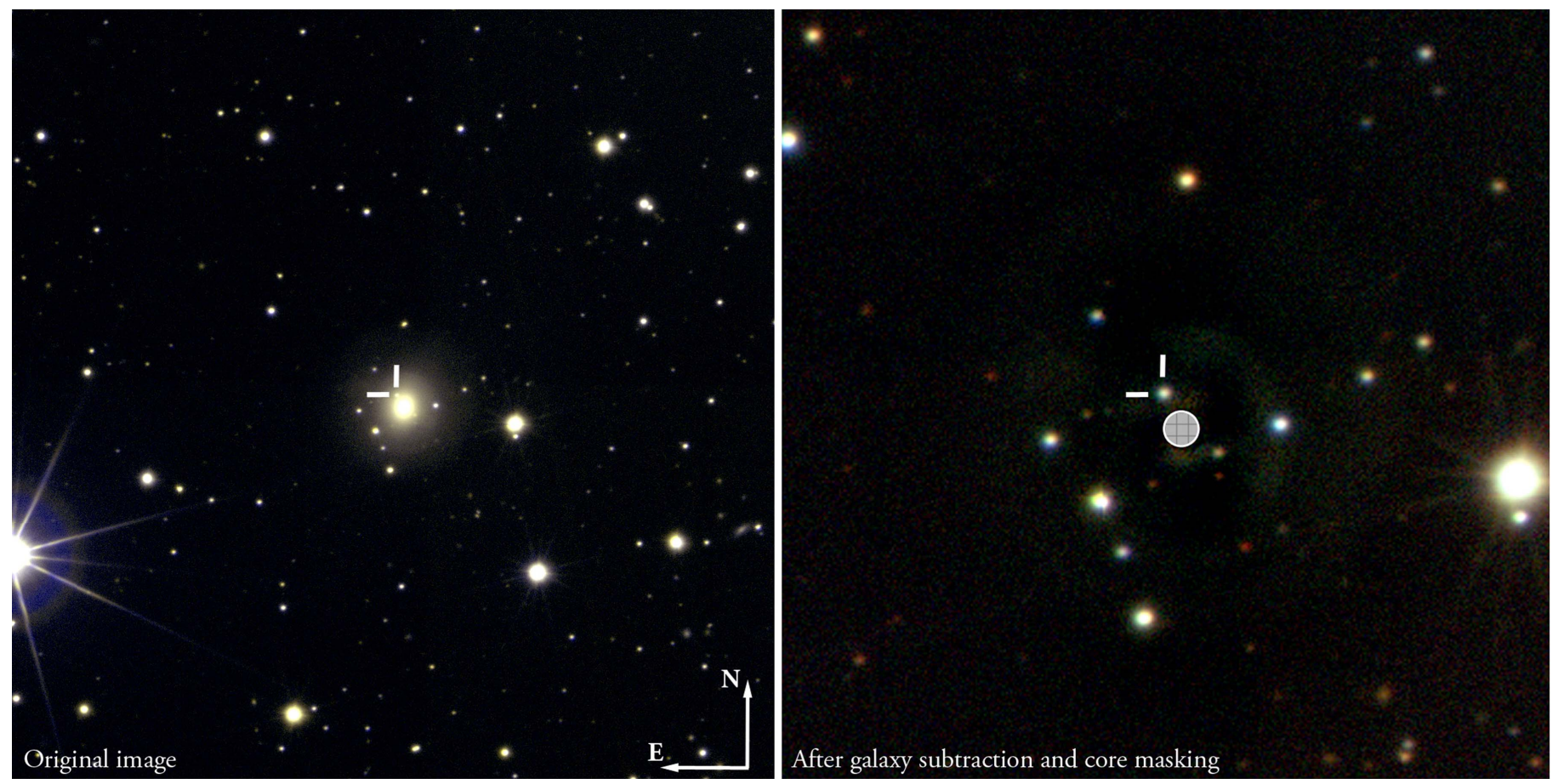

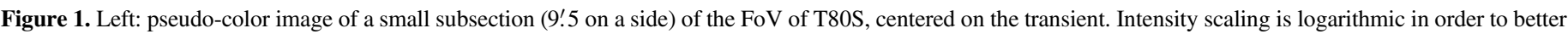

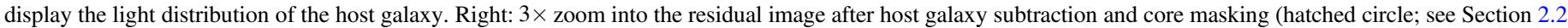
for details).

of the galaxy center was already well constrained by the initial PSF photometry. The right panel of Figure 1 shows the outcome of this procedure. Removing the host galaxy improved the determination of the local sky value and therefore reduced the uncertainty in the PSF magnitudes of stars in its vicinity by factors of $\sim 1.5,2.2$ and 2.0 in gri, respectively.

We performed the aforementioned procedures with DAOPHOT and ALLSTAR on the galaxy-subtracted master frames and generated "master star lists" for further analysis. The master star lists were used as input for ALLFRAME to perform simultaneous PSF photometry on all of the host galaxy-subtracted images obtained with T80S in a given filter (due to the very low signalto-noise ratio of the individual EABA images, we only obtained a single measurement for this data set using its master frame and did not perform time-series photometry). We identified 100 bright and isolated stars in each filter within the T80S FoV and used them to solve for frame-to-frame zeropoint offsets, in order to correct for differential extinction and any other variations. We achieved a photometric precision in our T80S time-series photometry of $0.01 \mathrm{mag}$ or better for objects with $g<16$, $r<15, i<14$ mag.

We transformed the T80S measurements into the PanSTARRS1 photometric system (Tonry et al. 2012) and simultaneously corrected for atmospheric extinction using 4600-5400 objects in common between our star lists and the PS1 catalog available at the Mikulski Archive for Space Telescopes (MAST). ${ }^{24}$ We used the PS1 PSF magnitudes for the transformations. The selected stars spanned $-0.6<g-i<3.2$, enabling us to solve for a quadratic

\footnotetext{
${ }^{24}$ MAST is part of STScI, operated by AURA, Inc., under NASA contract NAS5-26555. Support for MAST for non-HST data is provided by the NASA Office of Space Science via grant NNX09AF08G and by other grants and contracts.
}

transformation in each band as a function of that color. We found small but statistically significant color terms for all of the transformations, with residual dispersions of $0.03 \mathrm{mag}$.

The calibrated light curves are plotted in Figure 2 and the time-series photometry is presented in Table $1 .{ }^{25}$ The tabulated uncertainties were calculated by ALLFRAME and related programs based on the PSF fitting results and the frameto-frame zeropoint corrections. We present the analysis of the light curves in Section 3.

We calibrated the EABA $1.5 \mathrm{~m}$ observations in a similar manner. Due to the significantly smaller FoV and worse image quality, we were limited to 200 stars in common. Since these images were obtained without a filter, we solved for a linear transformation with respect to $r$, which exhibited a very large $r-i$ color term $(-0.54 \pm 0.03)$.

\section{Analysis}

The T80S light curve of the transient exhibits a very significant decline across all bands during the $\sim 80$ minutes of observations. A weighted linear fit to the data yields $\Delta g=0.17 \pm 0.03 \mathrm{mag}$, $\Delta r=0.14 \pm 0.02 \mathrm{mag}, \Delta i=0.10 \pm 0.03 \mathrm{mag}$ over that time period. The mean magnitudes at the mid-point of our observations (1.467 days after the $\mathrm{GW}$ trigger) and their time derivatives (expressed in magnitudes per day) are as follows:

$$
\begin{array}{rlrl}
g & =18.60 \pm 0.02 \mathrm{mag}, & & d g / d t=3.0 \pm 0.6 \mathrm{mag} / \text { day } \\
r & =17.99 \pm 0.02, & & d r / d t=2.5 \pm 0.4 \\
i=17.80 \pm 0.02, & & d i / d t=1.9 \pm 0.5 .
\end{array}
$$

We ruled out systematic effects as the reason for the fast decline by examining the light curves of all 885 objects in the

\footnotetext{
25 Only a portion of the Table is shown here for guidance and context. The full version is available online.
} 


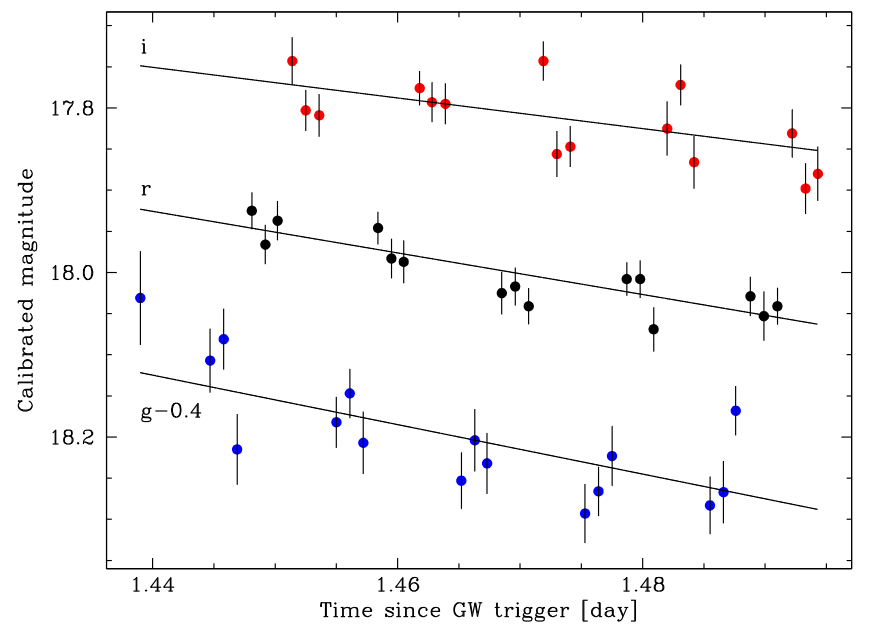

Figure 2. gri light curves of the EM counterpart to GW170817, obtained with T80S on 2017 August 18. The $g$ points have been offset by -0.4 mag for clarity.

Table 1

Time-series Photometry

\begin{tabular}{lccc}
\hline \hline Time $^{\mathrm{a}}$ & Band & Mag & $\sigma(\mathrm{mag})$ \\
\hline 1.4390 & $g$ & 18.43 & 0.06 \\
1.4447 & $g$ & 18.51 & 0.04 \\
1.4458 & $g$ & 18.48 & 0.04 \\
1.4469 & $g$ & 18.62 & 0.04 \\
1.4481 & $r$ & 17.93 & 0.02 \\
1.4492 & $r$ & 17.97 & 0.02 \\
1.4502 & $r$ & 17.94 & 0.02 \\
1.4514 & $i$ & 17.74 & 0.03 \\
\hline
\end{tabular}

Note.

${ }^{a}$ Days since GW trigger.

(This table is available in its entirety in machine-readable form.)

T80S FoV with similar colors to the transient (within $0.1 \mathrm{mag}$ in both $g-r$ and $r-i$ ). We performed weighted linear fits as a function of time on the light curves of all of the selected objects in each band, and estimated the statistical significance of the first-order coefficient (hereafter, "slope"). The results are shown as histograms in the top half of Figure 3. It can be seen that the distributions are approximately centered on zero and that the transient exhibits some of the fastest recorded decline rates in all bands. The bottom panels of Figure 3 show that most of the light curve slopes for the objects in the comparison sample are not statistically significant, while the decline rates for the transient are among the most highly statistically significant.

As we do not have a measurement of the $r-i$ color of the transient during the EABA observations, we present two possible values for the calibrated magnitude of the object at that time (2.456 days after the trigger): $r=18.78 \pm 0.03$ mag if its color did not evolve relative to the previous night $(r-i=0.19 \pm 0.03 \mathrm{mag})$, or $r=19.15 \pm 0.06 \mathrm{mag}$ if its color evolved as extrapolated from the T80S light curve to $r-i \sim 0.84 \pm 0.08$ mag. Despite the limited information obtained from EABA, these observations confirm a fast decline in luminosity of $\Delta r \sim 0.8-1.2$ mag over $24 \mathrm{hr}$.

Figure 4 compares our photometry (adjusted to $D=38 \mathrm{Mpc}$ ) with the gri light curves predicted by three kilonova models (see Figure 8 of Tanaka et al. 2017, hereafter T17), which build upon
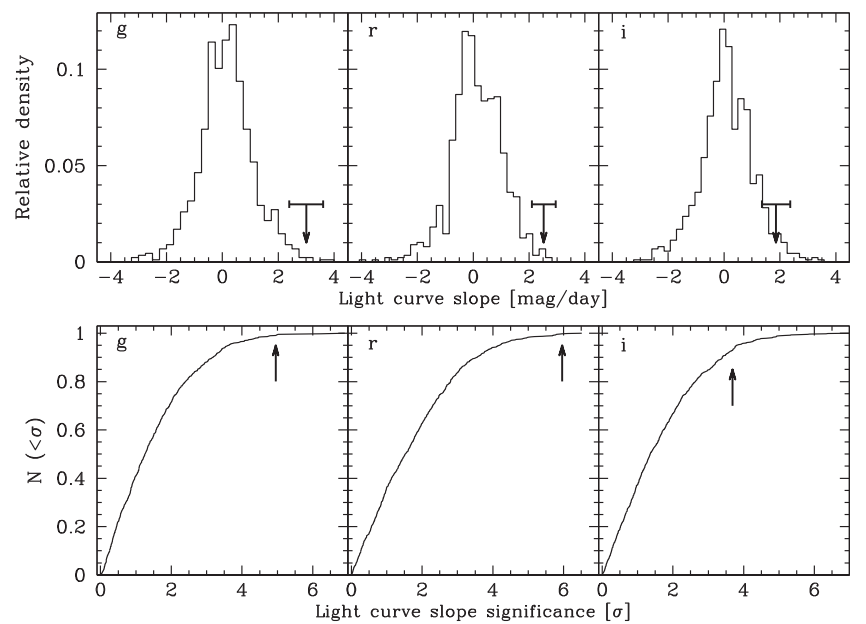

Figure 3. Top: comparison of the light curve slopes (magnitudes per day) of the transient (arrows) in gri (left, center, and right panels, respectively) relative to 885 objects in the T80S FoV with similar colors $(|g-r|,|r-i|<0.1)$. The horizontal error bars indicate the $1 \sigma$ uncertainty in the values for the transient. Bottom: statistical significance of the values for the transient in gri (left, center, and right panels, respectively) relative to the comparison sample.

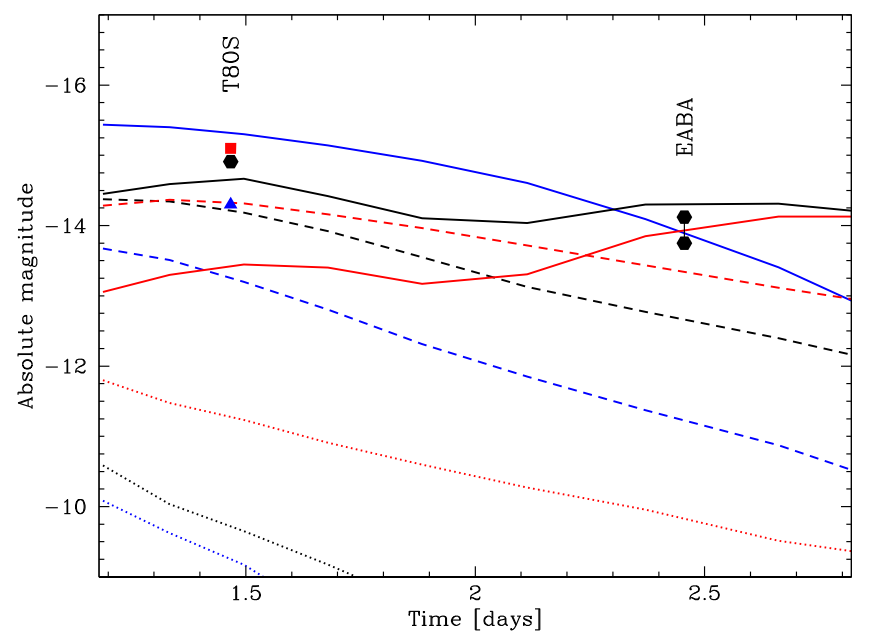

Figure 4. Comparison of our photometry ( $g$ : blue triangle; $r$ : black hexagons; $i$ : red square) adjusted to $D=38 \mathrm{Mpc}$ with models from Tanaka et al. (2017) plotted using the same color scheme. The dotted lines represent a "red kilonova" model with dynamical ejecta rich in lanthanides. The dashed and solid lines represent "blue kilonova" wind models with decreasing amounts of lanthanides. The measurement uncertainties are smaller than the size of the symbols. The two possible $r$-values at 2.456 days are discussed in Section 3 .

the work of Tanaka \& Hotokezaka (2013). We refer interested readers to those publications for details of the parameters used in each model.

Our absolute magnitudes and colors are inconsistent with the predictions of a "red kilonova" model containing dynamical ejecta $\left(0.01 M_{\odot}, v=0.2 c\right)$ rich in lanthanides (dotted lines). Our $r$-band luminosity is in fairly good agreement with the prediction of a "blue kilonova" model with a "wind" $\left(0.01 M_{\odot}\right.$, $v=0.05 c)$ free of lanthanides $\left(Y_{e}=0.3\right.$ variant in $\mathrm{T} 17$, solid lines), but the predicted $g-i$ color of this model at $t=1.5$ days does not match the observations. A similar "wind model" $\left(0.01 M_{\odot}, v=0.05 c\right)$, in which the ejecta contain a small amount of lanthanides ( $Y_{e}=0.25$ variant in T17, dashed lines), matches the observed colors fairly well, but the predicted luminosities are somewhat lower than observed. Regardless, it 
appears that this event may have been a "blue kilonova" (Metzger 2017) relatively free of lanthanides.

Intriguingly, there is a low-significance $(\sim 0.1 \mathrm{mag})$ modulation in the T80S light curve residuals across all bands. If confirmed by others, such a correlated departure from a linear decay may be useful for discriminating among models. As seen in Figure 4, the light curves predicted by the two "blue" kilonova models exhibit quite different behavior at the time of our observations.

\section{Concluding Remarks}

The detection of a merger of two neutron stars by Advanced LIGO/Virgo has opened a new chapter of GW astronomy. The combination of three $\mathrm{GW}$ interferometers provided a robust and relatively small localization region for the event, and the prompt follow-up and sharing of information by many collaborations resulted in a timely identification of its EM counterpart.

Given the fast temporal evolution of these transients, it is clear that continued worldwide follow-up efforts will be critical for providing early and nearly continuous coverage, minimizing weather losses, and maximizing the astrophysical constraints that can be extracted from photometric and spectroscopic observations.

We thank the referee for a very prompt and helpful report and Dr. Masaomi Tanaka for kindly sharing his latest kilonova models in advance of publication. M.D. acknowledges NSF support through grant NSF-HRD 1242090. D.G.L. and the IATE/UNC team acknowledge support from the Consejo Nacional de Investigaciones Científicas y Técnicas of Argentina. C.Md.O. and the S-PLUS team are thankful to FAPESP (grant 2009/54202-8) for funding the T80-South robotic telescope and its camera, to the Observatorio Nacional-MCT for funding the T80-South building, to INPE for help with the design of the camera, and to the staff of CEFCA for constant support over the years in the design and testing of T80S and its data reduction pipeline. J.L.N.C. is grateful for financial support from the Southern Office of Aerospace Research and Development (SOARD), a branch of the Air Force Office of the Scientific Research International Office of the United States (AFOSR/IO), through grant FA9550-15-1-0167. J.L.N.C. also acknowledges financial support from the Dirección de Investigación y Desarrollo de la Universidad de La Serena through the Programa de Incentivo a la Investigación Académica (PIA-DIULS). R.L.O. was supported by the Brazilian agency CNPq (PDE-200289/ 2017-9 and universal-459553/2014-3). S.T.F. acknowledges financial support from the Universidad of La Serena for funding two technician positions for the T80S project. Finally, the entire TOROS Collaboration would like to thank the Argentine Gemini TAC and the Mexican GTC TAC for awarding ToO time to TOROS during the first semester of 2017.

\section{ORCID iDs}

M. C. Díaz (ํ) https://orcid.org/0000-0002-7555-8856

L. M. Macri (1) https://orcid.org/0000-0002-1775-4859

V. Chavushyan (1) https://orcid.org/0000-0002-2558-0967

D. Dultzin (i) https://orcid.org/0000-0001-5756-8842

O. López-Cruz (i) https://orcid.org/0000-0002-1381-7437

J. L. Marshall ๑ https://orcid.org/0000-0003-0710-9474

N. Padilla 누 https://orcid.org/0000-0001-9850-9419

C. Quiñones ㄴ) https://orcid.org/0000-0003-0276-9879

E. Ríos-López i https://orcid.org/0000-0002-4436-221X

\section{References}

Abbott, B. P., Abbott, R., Abbott, T. D., et al. 2016a, PhRvL, 116, 061102 Abbott, B. P., Abbott, R., Abbott, T. D., et al. 2016b, PhRvL, 116, 241103 Abbott, B. P., Abbott, R., Abbott, T. D., et al. 2017a, PhRvL, 118, 221101

Abbott, B. P., Abbott, R., Abbott, T. D., et al. 2017b, PhRvL, https://doi.org/ 10.1103/PhysRevLett.119.141101

Acernese, F., Agathos, M., Agatsuma, K., et al. 2015, CQGra, 32, 024001

Allam, S., Annis, J., Berger, E., et al. 2017, GCN, 21530

Allen, B., Anderson, W. G., Brady, P. R., Brown, D. A., \& Creighton, J. D. E. 2012, PhRvD, 85, 122006

Benacquista, M., Belczynski, C., Beroiz, M., et al. 2014, in The Milky Way Unravelled by Gaia: GREAT Science from the Gaia Data Releases, EAS Publications Ser. 67, ed. N. A. Walton et al. (London: EDP Sciences), 357

Bissaldi, E., von Kienlin, A., Lichti, G., et al. 2009, ExA, 24, 47

Chen, H.-Y., Holz, D. E., Miller, J., et al. 2017, arXiv:1709.08079

Colazo, C., Lambas, D. G., Sanchez, B., et al. 2015, GCN, 18338

Connaughton, V., Blackburn, L., Briggs, M. S., et al. 2017, GCN, 21506

Coulter, D. A., Kilpatrick, C. D., Siebert, M. R., et al. 2017, GCN, 21529

Díaz, M. C., Benacquista, M., Belczynski, K., et al. 2014, in The Third Hot-wiring the Transient Universe Workshop, ed. P. R. Wozniak et al. (Menlo Park, CA: SLAC), 225, http://www.slac.stanford.edu/econf/C131113.1/papers/diaz.pdf Díaz, M. C., Beroiz, M., Peñuela, T., et al. 2016, ApJL, 828, L16

Díaz, M. C., Garcia Lambas, D., Macri, L. M., et al. 2017a, GCN, 21619

Díaz, M. C., Garcia Lambas, D., Macri, L. M., et al. 2017b, GCN, 21620

Erwin, P. 2015, ApJ, 799, 226

Finn, L. S., \& Chernoff, D. F. 1993, PhRvD, 47, 2198

Goldstein, A., Veres, P., von Kienlin, A., et al. 2017, GCN, 21528

Hulse, R. A., \& Taylor, J. H. 1975, ApJL, 195, L51

Kourkchi, E., \& Tully, R. B. 2017, ApJ, 843, 16

LIGO Scientific Collaboration \& Virgo Collaboration 2017a, GCN, 21505

LIGO Scientific Collaboration \& Virgo Collaboration 2017b, GCN, 21509

LIGO Scientific Collaboration \& Virgo Collaboration 2017c, GCN, 21510

LIGO Scientific Collaboration \& Virgo Collaboration 2017d, GCN, 21513

LIGO Scientific Collaboration \& Virgo Collaboration 2017e, GCN, 21527

LIGO Scientific Collaboration \& Virgo Collaboration 2017f, PhRvL, https:// doi.org/10.1103PhysRevLett.119.161101

LIGO Scientific Collaboration 2015, CQGra, 32, 074001

Macri, L. M., Ngeow, C.-C., Kanbur, S. M., Mahzooni, S., \& Smitka, M. T. 2015, AJ, 149, 117

Macri, L. M., Stanek, K. Z., Bersier, D., Greenhill, L. J., \& Reid, M. J. 2006, ApJ, 652, 1133

Metzger, B. D. 2017, LRR, 20, 3

Renzi, V., Vrech, R., Ferreiro, D., et al. 2009, BAAA, 52, 285

Stetson, P. B. 1987, PASP, 99, 191

Stetson, P. B. 1994, PASP, 106, 250

Tanaka, M., \& Hotokezaka, K. 2013, ApJ, 775, 113

Tanaka, M., Kato, D., Gaigalas, G., et al. 2017, arXiv:1708.09101

Taylor, J. H., \& Weisberg, J. M. 1982, ApJ, 253, 908

Tonry, J. L., Stubbs, C. W., Lykke, K. R., et al. 2012, ApJ, 750, 99

Tremblin, P., Schneider, N., Minier, V., Durand, G. A., \& Urban, J. 2012, A\&A, 548, A65

von Kienlin, A., Meegan, C., Goldstein, A., et al. 2017, GCN, 21520

Yang, S., Valenti, S., Sand, D., et al. 2017, GCN, 21531 